\title{
WEAK SYMMETRIC INTEGRALS WITH RESPECT TO THE FRACTIONAL BROWNIAN MOTION
}

\author{
By GiUlia BinOtTo ${ }^{1}$, IVAN NOURdin ${ }^{2}$ AND DAVID NuALART ${ }^{3}$ \\ Universitat de Barcelona, Université du Luxembourg and University of Kansas
}

The aim of this paper is to establish the weak convergence, in the topology of the Skorohod space, of the $v$-symmetric Riemann sums for functionals of the fractional Brownian motion when the Hurst parameter takes the critical value $H=(4 \ell+2)^{-1}$, where $\ell=\ell(v) \geq 1$ is the largest natural number satisfying $\int_{0}^{1} \alpha^{2 j} v(d \alpha)=\frac{1}{2 j+1}$ for all $j=0, \ldots, \ell-1$. As a consequence, we derive a change-of-variable formula in distribution, where the correction term is a stochastic integral with respect to a Brownian motion that is independent of the fractional Brownian motion.

1. Introduction. Suppose that $B^{H}=\left\{B_{t}^{H}, t \geq 0\right\}$ is a fractional Brownian motion (fBm) with Hurst parameter $H \in(0,1)$, that is, $B^{H}$ is a centered Gaussian process with covariance given by

$$
R(s, t):=\mathbb{E}\left[B_{s}^{H} B_{t}^{H}\right]=\frac{1}{2}\left(s^{2 H}+t^{2 H}-|t-s|^{2 H}\right),
$$

for any $s, t \geq 0$. When $H<\frac{1}{2}$, it is well known that the integral $\int_{0}^{t} g\left(B_{s}^{H}\right) d B_{s}^{H}$ does not exist in general as a path-wise Riemann-Stieltjes integral. In the pioneering work [5], Gradinaru, Nourdin, Russo and Vallois proved that this integral can be defined as the limit in probability of suitable symmetric Riemann sums if the Hurst parameter is not too small. Let us briefly describe the main contribution of $[5]$.

Let $v$ be a symmetric probability measure on $[0,1]$, meaning that $v(A)=v(1-$ $A$ ) for any Borel set $A \subset[0,1]$. Given a continuous function $g: \mathbb{R} \rightarrow \mathbb{R}$, consider the $v$-symmetric Riemann sums of $g\left(B_{s}^{H}\right)$ in the interval $[0, t]$ given by

$$
S_{n}^{\nu}(g, t)=\sum_{j=0}^{\lfloor n t\rfloor-1}\left(B_{\frac{j+1}{n}}^{H}-B_{\frac{j}{n}}^{H}\right) \int_{0}^{1} g\left(B_{\frac{j}{n}}^{H}+\alpha\left(B_{\frac{j+1}{n}}^{H}-B_{\frac{j}{n}}^{H}\right)\right) v(d \alpha),
$$

Received June 2016; revised July 2017.

${ }^{1}$ Supported by the Grant MTM2012-31192 from SEIDI, Ministerio de Economia y Competividad.

${ }^{2}$ Supported in part by the Grant F1R-MTH-PUL-15CONF (CONFLUENT) at Luxembourg University.

${ }^{3}$ Supported by NSF Grant DMS-15-12891 and ARO Grant FED0070445.

MSC2010 subject classifications. 60G05, 60H07, 60G15, 60F17.

Key words and phrases. Fractional Brownian motion, Stratonovich integrals, Malliavin calculus, Itô formula in law. 
where $n \geq 1$ is an integer and $\lfloor x\rfloor$ denotes the integer part of $x$ for any $x \geq 0$. Then, following [5] and providing the limit exists, the $v$-symmetric integral is defined as the limit in probability of the $v$-symmetric Riemann sums as $n$ tends to infinity, namely,

$$
\int_{0}^{t} g\left(B_{s}^{H}\right) d^{v} B_{s}^{H}=\lim _{n \rightarrow \infty} S_{n}^{\nu}(g, t) .
$$

It is proved in [5] that this integral exists for $g=f^{\prime}$ with $f \in \mathcal{C}^{4 \ell(v)+2}(\mathbb{R})$, if the Hurst parameter satisfies $H>\frac{1}{4 \ell(v)+2}$. Here, we denote by $\ell(v) \geq 1$ the largest positive natural number such that

$$
\int_{0}^{1} \alpha^{2 j} v(d \alpha)=\frac{1}{2 j+1} \quad \forall j=0,1, \ldots, \ell(v)-1 .
$$

Moreover, in this case the integral $\int_{0}^{t} f^{\prime}\left(B_{s}^{H}\right) d^{v} B_{s}^{H}$ satisfies the chain rule

$$
f\left(B_{t}^{H}\right)=f(0)+\int_{0}^{t} f^{\prime}\left(B_{s}^{H}\right) d^{v} B_{s}^{H} .
$$

Basic examples of $v$-symmetric Riemann sums and integrals are the following:

(i) If $v=\frac{1}{2} \delta_{0}+\frac{1}{2} \delta_{1}$, then $S_{n}^{v}$ are the trapezoidal Riemann sums. In this case, $\ell(\nu)=1$ and $\nu$-symmetric integrals exist for $H>\frac{1}{6}$.

(ii) If $v=\frac{1}{6} \delta_{0}+\frac{2}{3} \delta_{1 / 2}+\frac{1}{6} \delta_{1}$, then $\ell(v)=2$. In this case, $S_{n}^{v}$ are the SimpsonRiemann sums and $\nu$-symmetric integrals exist for $H>\frac{1}{10}$.

(iii) If $v$ is the Lebesgue measure, then $\ell(v)=\infty$, and $v$-symmetric integrals exist for any $H \in(0,1)$.

The lower bound $\frac{1}{4 \ell(v)+2}$ for the Hurst parameter is sharp, in the sense that for $H=\frac{1}{4 \ell(v)+2}$ the $v$-symmetric integral diverges in $L^{2}(\Omega)$ for $f(x)=x^{2}$. This has been proved, for the example (i) above, in the references [2] and [5]. The goal of this paper is to show that when $H=\frac{1}{4 \ell(v)+2}$, the $v$-symmetric Riemann sums converge in distribution and, as a consequence, we obtain a change-of-variable formula in law with a correction term which is an Itô stochastic integral with respect to a Brownian motion which is independent of $B^{H}$. More precisely, the main result is the following theorem.

We say that a function $f: \mathbb{R} \rightarrow \mathbb{R}$ has moderate growth if there exist positive constants $A, B$ and $\alpha<2$ such that $|f(x)| \leq A e^{B|x|^{\alpha}}$ for all $x \in \mathbb{R}$.

THEOREM 1.1. Fix a symmetric probability measure $v$ on $[0,1]$ with $\ell:=$ $\ell(v)<\infty$ and let $B^{H}=\left\{B_{t}^{H}, t \geq 0\right\}$ be a fractional Brownian motion with Hurst parameter $H=\frac{1}{4 \ell+2}$. Consider a function $f \in \mathcal{C}^{20 \ell+5}(\mathbb{R})$ such that $f$ and its derivatives up to the order $20 \ell+5$ have moderate growth. Then

$$
S_{n}^{\nu}\left(f^{\prime}, t\right) \underset{n \rightarrow \infty}{\stackrel{\mathcal{L}}{\longrightarrow}} f\left(B_{t}^{H}\right)-f(0)-c_{\nu} \int_{0}^{t} f^{(2 \ell+1)}\left(B_{s}^{H}\right) d W_{s},
$$


where $W=\left\{W_{t}, t \geq 0\right\}$ is a Brownian motion independent of $B^{H}, c_{v}$ is a constant depending only on $v$ and the convergence holds in the topology of the Skorohod space $D([0, \infty))$.

The value of the constant $c_{\nu}$ in (1.3) is $c_{v}=k_{v, \ell} \sigma_{\ell}$, where $k_{v, \ell}$ is defined in (3.2) and $\sigma_{\ell}$ is given by

$$
\sigma_{\ell}^{2}=\mathbb{E}\left[X_{1}^{4 \ell+2}\right]+2 \sum_{j=1}^{\infty} \mathbb{E}\left[\left(X_{1} X_{1+j}\right)^{2 \ell+1}\right],
$$

where $X_{j}=B_{j}^{1 /(4 \ell+2)}-B_{j-1}^{1 /(4 \ell+2)}$ for $j \geq 1$ (see, for instance, [14], Theorem 10$)$.

The statement of Theorem 1.1 can be interpreted as a change-of-variable formula in law. Indeed, although the sequence of $v$-symmetric Riemann sums $S_{n}^{\nu}\left(f^{\prime}, t\right)$ fails in general to converge in probability and the $v$-symmetric integral $\int_{0}^{t} f^{\prime}\left(B_{s}^{H}\right) d^{v} B_{s}^{H}$ does not exist in the sense introduced above, this sequence converges in law and we can still call the limit (which is defined only in law) the $v$-symmetric integral, and denote it by $\int_{0}^{t} f^{\prime}\left(B_{s}^{H}\right) d^{v} B_{s}^{H}$. In this way, we can write

$$
f\left(B_{t}^{H}\right)=f(0)+\int_{0}^{t} f^{\prime}\left(B_{s}^{H}\right) d^{v} B_{s}^{H}+c_{v} \int_{0}^{t} f^{(2 \ell+1)}\left(B_{s}^{H}\right) d W_{s},
$$

where this formula has to be understood in the sense that the random variables $\int_{0}^{t} f^{\prime}\left(B_{s}^{H}\right) d^{v} B_{s}^{H}$ and $f\left(B_{t}^{H}\right)-f(0)-c_{v} \int_{0}^{t} f^{(2 \ell+1)}\left(B_{s}^{H}\right) d W_{s}$ have the same law.

Some particular cases have already been addressed recently in the literature. In the case $v=\frac{1}{2} \delta_{0}+\frac{1}{2} \delta_{1}$ (trapezoidal Riemann sums), the critical value is $H=\frac{1}{6}$, and the corresponding version of Theorem 1.1 was proved by Nourdin, Réveillac and Swanson in [12]. The convergence results for trapezoidal Riemann sums were extended to a general class of Gaussian processes by Harnett and Nualart in [6]. If $v=\frac{1}{6} \delta_{0}+\frac{2}{3} \delta_{\frac{1}{2}}+\frac{1}{6} \delta_{1}$ (Simpson Riemann sums), the critical point is $H=\frac{1}{10}$ and the convergence in law for any fixed time $t \geq 0$ was proved by Harnett and Nualart in [8].

For related results in the case of midpoint Riemann sums, we refer to the works by Burdzy and Swanson [1], Nourdin and Réveillac [11] and Harnett and Nualart [7]. In this case, the critical value of the Hurst parameter is $H=\frac{1}{4}$, and the complementary term in the Itô formula involves a second derivative; see also Nourdin [9].

Let us briefly describe the strategy we will follow for the proof of Theorem 1.1. First, using Taylor's formula and the properties of the symmetric measure $v$ derived in [5], we determine the following decomposition for $v$-symmetric Riemann sums:

$$
S_{n}^{\nu}\left(f^{\prime}, t\right)=f\left(B_{\lfloor n t\rfloor}^{H}\right)-f(0)-\sum_{h=\ell}^{2 \ell} \Phi_{n}^{h}(t)-R_{n}(t),
$$


where, for each $h=\ell, \ldots, 2 \ell$,

$$
\Phi_{n}^{h}(t)=\sum_{j=0}^{\lfloor n t\rfloor-1} k_{\nu, h} f^{(2 h+1)}\left(\widetilde{B}_{\frac{j}{n}}^{H}\right)\left(\Delta_{j}^{n} B^{H}\right)^{2 h+1},
$$

the constants $k_{\nu, h}$ are defined in (3.2) and we use the notation $\widetilde{B}_{\frac{j}{n}}^{H}=\frac{1}{2}\left(B_{\frac{j}{n}}^{H}+B_{\frac{j+1}{n}}^{H}\right)$ and $\Delta_{j}^{n} B^{H}=B_{\frac{j+1}{n}}^{H}-B_{\frac{j}{n}}^{H}$. The residual term $R_{n}(t)$ is a weighted sum of the powers $\left(\Delta_{j}^{n} B^{H}\right)^{4 \ell+2}$ with coefficients that converge to zero as $n$ tends to infinity. Taking into account that $H=\frac{1}{4 \ell+2}$, it is not difficult to show that $R_{n}(t)$ converges to zero in probability, uniformly in compact sets.

In Lemma A.1, proved in the Appendix, we show that for any $h=\ell, \ldots, 2 \ell$, the moment of order four $\mathbb{E}\left[\left|\Phi_{n}^{h}(t)-\Phi_{n}^{h}(s)\right|^{4}\right]$, for any $0 \leq s \leq t \leq T$, can be estimated by

$$
C_{T} \sum_{N=2}^{4} n^{\frac{2(\ell-h) N}{2 \ell+1}}\left(\frac{\lfloor n t\rfloor-\lfloor n s\rfloor}{n}\right)^{N} .
$$

This lemma is proved by expressing the product of increments $\prod_{i=1}^{4}\left(\Delta_{j_{i}}^{n} B^{H}\right)^{2 h+1}$ as a linear combination of multiple stochastic integrals and applying the duality relationship between multiple stochastic integrals and the iterated Malliavin derivative. As a consequence, for $h=\ell+1, \ldots, 2 \ell$, the terms $\Phi_{n}^{h}(t)$ converge to zero in the topology of the Skorohod space $D([0, \infty))$, and for $h=\ell$, the sequence $\Phi_{n}^{\ell}(t)$ is tight.

Thus, the only nonzero contribution to the limit in law of the $v$-symmetric Riemann sums $S_{n}^{v}\left(f^{\prime}, t\right)$ is the term

$$
k_{\nu, \ell} \sum_{j=0}^{\lfloor n t\rfloor-1} f^{(2 \ell+1)}\left(\widetilde{B}_{\frac{j}{n}}^{H}\right)\left(\Delta_{j}^{n} B^{H}\right)^{2 \ell+1},
$$

and it suffices to show that the finite dimensional distributions of this process converge to those of $c_{v} \int_{0}^{t} f^{(2 \ell+1)}\left(B_{s}^{H}\right) d W_{s}$. Notice that the process appearing in (1.5) is a weighted sum of the odd powers $\left(\Delta_{j}^{n} B^{H}\right)^{2 \ell+1}$ of the fBm. It is well known that for $H=\frac{1}{4 \ell+2}$, the sums of these odd powers converge in law to a Gaussian random variable. More precisely, the following stable convergence holds:

$$
\left(\sum_{j=0}^{\lfloor n t\rfloor-1}\left(\Delta_{j}^{n} B^{H}\right)^{2 \ell+1}, B_{t}^{H}, t \geq 0\right) \underset{n \rightarrow \infty}{\stackrel{\mathcal{L}}{\longrightarrow}}\left(\sigma_{\ell} W_{t}, B_{t}^{H}, t \geq 0\right),
$$

where $\sigma_{\ell}$ is defined in (1.4) and in the right-hand side, the process $W$ is a Brownian motion independent of $B^{H}$. The proof of the convergence for a fixed $t$ follows from the Breuer-Major theorem (we refer to [10], Chapter 7, and [4] for a proof of this result based on the fourth moment theorem). Then the convergence of the weighted 
sums (1.5) follows from the methodology of small blocks/big blocks used, for instance in the works [3] and [4]. The basic ingredient in this approach is the proof that the reminder term converges to zero and this follows from Lemma A.2, proved in the Appendix. However, unlike the above references, the convergence to zero of the reminder term cannot be established using fractional calculus techniques because $H<\frac{1}{2}$, and it requires the application of integration-by-parts formulas from Malliavin calculus.

The paper is organized as follows. Section 2 contains some preliminaries on the Malliavin calculus and the fractional Brownian motion. Section 3 is devoted to the proof of Theorem 1.1 and in the Appendix we show two basic technical lemmas.

2. Preliminaries. In the next two subsections, we discuss some notions of Malliavin calculus and fractional Brownian motion. Throughout the paper, $C_{T}$ and $C$ will denote any positive constants depending or not on $T$, respectively; they may change from one expression to another.

2.1. Elements of Malliavin calculus. Let $\mathfrak{H}$ be a real separable infinitedimensional Hilbert space and let $X=\{X(h), h \in \mathfrak{H}\}$ be an isonormal Gaussian process over $\mathfrak{H}$. This means that $X$ is a centered Gaussian family, defined on a complete probability space $(\Omega, \mathcal{F}, P)$, with a covariance structure given by

$$
\mathbb{E}[X(h) X(g)]=\langle h, g\rangle_{\mathfrak{H}}, \quad h, g \in \mathfrak{H} .
$$

We assume that $\mathcal{F}$ is the $\sigma$-algebra generated by $X$.

For any integer $q \geq 1$, let $\mathfrak{H}^{\otimes q}$ and $\mathfrak{H}^{\odot q}$ denote, respectively, the $q$ th tensor product and the $q$ th symmetric tensor product of $\mathfrak{H}$.

Let $\left\{e_{n}, n \geq 1\right\}$ be a complete orthonormal system in $\mathfrak{H}$. Given $f \in \mathfrak{H}^{\odot p}, g \in$ $\mathfrak{H}^{\odot q}$ and $r \in\{0, \ldots, p \wedge q\}$, the $r$ th-order contraction of $f$ and $g$ is the element of $\mathfrak{H}^{\otimes(p+q-2 r)}$ defined by

$$
f \otimes_{r} g=\sum_{i_{1}, \ldots, i_{r}=1}^{\infty}\left\langle f, e_{i_{1}} \otimes \cdots \otimes e_{i_{r}}\right\rangle_{\mathfrak{H}^{\otimes r}} \otimes\left\langle g, e_{i_{1}} \otimes \cdots \otimes e_{i_{r}}\right\rangle_{\mathfrak{H}^{\otimes r}},
$$

where $f \otimes_{0} g=f \otimes g$ and, for $p=q, f \otimes_{q} g=\langle f, g\rangle_{\mathfrak{H} \otimes q}$. Notice that $f \otimes_{r} g$ is not necessarily symmetric. We denote its symmetrization by $f \widetilde{\otimes}_{r} g \in \mathfrak{H}^{\odot(p+q-2 r)}$.

Let $\mathcal{H}_{q}$ denote the $q$ th Wiener chaos of $X$, that is, the closed linear subspace of $L^{2}(\Omega)$ generated by the random variables $\left\{H_{q}(X(h)), h \in \mathfrak{H},\|h\|_{\mathfrak{H}}=1\right\}$, where $H_{q}$ is the $q$ th Hermite polynomial defined by

$$
H_{q}(x)=(-1)^{q} e^{x^{2} / 2} \frac{d^{q}}{d x^{q}}\left(e^{-x^{2} / 2}\right) .
$$

For $q \geq 1$, let $I_{q}(\cdot)$ denote the generalized Wiener-Itô multiple stochastic integral. It is known that the map

$$
I_{q}\left(h^{\otimes q}\right)=H_{q}(X(h))
$$


provides a linear isometry between $\mathfrak{H}^{\odot q}$ (equipped with the modified norm $\sqrt{q !}\|\cdot\|_{\mathfrak{H} \otimes q}$ ) and $\mathcal{H}_{q}$ [equipped with the $L^{2}(\Omega)$ norm]. For $q=0$, we set by convention $\mathcal{H}_{0}=\mathbb{R}$ and $I_{0}$ equal to the identity map.

Multiple stochastic integrals satisfy the following product formula. Let $p, q \geq 1$ positive integers. Let $f \in \mathfrak{H}^{\odot p}$ and $g \in \mathfrak{H}^{\odot q}$. Then,

$$
I_{p}(f) I_{q}(g)=\sum_{z=0}^{p \wedge q} z !\left(\begin{array}{l}
p \\
z
\end{array}\right)\left(\begin{array}{l}
q \\
z
\end{array}\right) I_{p+q-2 z}\left(f \widetilde{\otimes}_{z} g\right),
$$

where $\otimes_{z}$ is the contraction operator defined in (2.1).

From the hypercontractivity property of the Ornstein-Uhlenbeck semigroup, it is well known that all $L^{r}(\Omega)$-norms, $r>1$, are equivalent on each Wiener chaos. In particular, for any real number $r \geq 2$, any integer $p \geq 2$ and any $f \in \mathfrak{H}^{\odot p}$, we have

$$
\left\|I_{p}(f)\right\|_{L^{r}(\Omega)} \leq C_{r, p}\left\|I_{p}(f)\right\|_{L^{2}(\Omega)}=C_{r, p} \sqrt{p !}\|f\|_{\mathfrak{H}^{\otimes p}} .
$$

Let $\mathcal{S}$ be the set of all smooth and cylindrical random variables of the form

$$
F=g\left(X\left(\phi_{1}\right), \ldots, X\left(\phi_{n}\right)\right),
$$

where $n \geq 1, g: \mathbb{R}^{n} \rightarrow \mathbb{R}$ is an infinitely differentiable function with compact support, and $\phi_{i} \in \mathfrak{H}$. The Malliavin derivative of $F$ with respect to $X$ is the element of $L^{2}(\Omega ; \mathfrak{H})$ defined as

$$
D F=\sum_{i=1}^{n} \frac{\partial g}{\partial x_{i}}\left(X\left(\phi_{1}\right), \ldots, X\left(\phi_{n}\right)\right) \phi_{i} .
$$

By iteration, we can define the $q$ th derivative $D^{q} F$ for every $q \geq 2$, which is an element of $L^{2}\left(\Omega ; \mathfrak{H}^{\odot q}\right)$.

For any integer $q \geq 1$ and any real number $p \geq 1$, let $\mathbb{D}^{q, p}$ denote the closure of $\mathcal{S}$ with respect to the norm $\|\cdot\|_{\mathbb{D} q, p}$, defined as

$$
\|F\|_{\mathbb{D}^{q, p}}^{p}=\mathbb{E}\left[|F|^{p}\right]+\sum_{i=1}^{q} \mathbb{E}\left(\left\|D^{i} F\right\|_{\mathfrak{H}^{\otimes i}}^{p}\right) .
$$

More generally, for any Hilbert space $V$, we denote by $\mathbb{D}^{q, p}(V)$ the corresponding Sobolev space of $V$-valued random variables.

The Malliavin derivative $D$ fulfills the following chain rule. If $\varphi: \mathbb{R}^{n} \rightarrow$ $\mathbb{R}$ is continuously differentiable with bounded partial derivatives and if $F=$ $\left(F_{1}, \ldots, F_{n}\right)$ is a vector of elements of $\mathbb{D}^{1,2}$, then $\varphi(F) \in \mathbb{D}^{1,2}$ and

$$
D \varphi(F)=\sum_{i=1}^{n} \frac{\partial \varphi}{\partial x_{i}}(F) D F_{i} .
$$

We denote by $\delta$ the Skorohod integral, also called the divergence operator, which is the adjoint of the operator $D$. More precisely, a random element $u \in$ 
$L^{2}(\Omega ; \mathfrak{H})$ belongs to the domain of $\delta$, denoted by Dom $\delta$, if and only if, for any $F \in \mathbb{D}^{1,2}$, we have

$$
\left|\mathbb{E}\left(\langle D F, u\rangle_{\mathfrak{H}}\right)\right| \leq c_{u}\|F\|_{L^{2}(\Omega)},
$$

where $c_{u}$ is a constant depending only on $u$. If $u \in \operatorname{Dom} \delta$, then the random variable $\delta(u)$ is defined by the duality relationship

$$
\mathbb{E}(F \delta(u))=\mathbb{E}\left(\langle D F, u\rangle_{\mathfrak{H}}\right) .
$$

This is called the Malliavin integration by parts formula and it holds for every $F \in \mathbb{D}^{1,2}$. For $q \geq 1$, the multiple Skorohod integral is defined iteratively as $\delta^{q}(u)=\delta\left(\delta^{q-1}(u)\right)$, with $\delta^{0}(u)=u$. From this definition, we have

$$
\mathbb{E}\left(F \delta^{q}(u)\right)=\mathbb{E}\left(\left\langle D^{q} F, u\right\rangle_{\mathfrak{H}^{\otimes q}}\right),
$$

for any $u \in \operatorname{Dom} \delta^{q}$ and any $F \in \mathbb{D}^{q, 2}$. Moreover, $\delta^{q}(h)=I_{q}(h)$ for any $h \in \mathfrak{H}^{\odot q}$. We refer to [13] for a detailed account on the Malliavin calculus for an arbitrary isonormal Gaussian process.

2.2. Fractional Brownian motion. Let $B^{H}=\left\{B_{t}^{H}, t \geq 0\right\}$ denote a fractional Brownian motion with Hurst parameter $H$. Namely, $B^{H}$ is a centered Gaussian process, defined on a complete probability space $(\Omega, \mathcal{F}, P)$ with covariance given by (1.1). We assume that $\mathcal{F}$ is generated by $B^{H}$. Along the paper, we suppose that $H<\frac{1}{2}$.

We denote by $\mathcal{E}$ the set of $\mathbb{R}$-valued step functions on $[0, \infty)$. Let $\mathfrak{H}$ be the Hilbert space defined as the completion of $\mathcal{E}$ with respect to the scalar product

$$
\left\langle\mathbf{1}_{[0, t]}, \mathbf{1}_{[0, s]}\right\rangle_{\mathfrak{H}}=R(s, t) .
$$

The mapping $\mathbb{1}_{[0, t]} \rightarrow B_{t}^{H}$ can be extended to a linear isometry between the Hilbert space $\mathfrak{H}$ and the Gaussian space spanned by $B^{H}$. In this way, $\left\{B^{H}(h), h \in \mathfrak{H}\right\}$ is an isonormal Gaussian process as in Section 2.1. we set

Recall the notation $\widetilde{B}_{\frac{j}{n}}^{H}=\frac{1}{2}\left(B_{\frac{j}{n}}^{H}+B_{\frac{j+1}{n}}^{H}\right)$ and $\Delta_{j}^{n} B^{H}=B_{\frac{j+1}{n}}^{H}-B_{\frac{j}{n}}^{H}$. Moreover,

$$
\begin{aligned}
\partial_{\frac{j}{n}} & =\mathbb{1}_{\left[\frac{j}{n}, \frac{j+1}{n}\right]}, \\
\varepsilon_{t} & =\mathbb{1}_{[0, t]}
\end{aligned}
$$

and

$$
\widetilde{\varepsilon}_{\frac{j}{n}}=\frac{1}{2}\left(\varepsilon_{\frac{j}{n}}+\varepsilon_{\frac{j+1}{n}}\right)=\frac{1}{2}\left(\mathbb{1}_{\left[0, \frac{j}{n}\right]}+\mathbb{1}_{\left[0, \frac{j+1}{n}\right]}\right) .
$$

The fractional Brownian motion with Hurst parameter $H$ satisfies

$$
\mathbb{E}\left[\left(\Delta_{j}^{n} B^{H}\right)^{2}\right]=\left\langle\partial_{\frac{j}{n}}, \partial_{\frac{j}{n}}\right\rangle_{\mathfrak{H}}=n^{-2 H} .
$$


Moreover, using the fact that the function $x \rightarrow x^{2 H}$ is concave for $H<\frac{1}{2}$, for any $t \geq 0$ and any integer $j \geq 0$, we obtain

$$
\left|\mathbb{E}\left[\left(\Delta_{j}^{n} B^{H}\right) B_{t}^{H}\right]\right|=\left|\left\langle\partial_{\frac{j}{n}}, \varepsilon_{t}\right\rangle_{\mathfrak{H}}\right| \leq n^{-2 H} .
$$

The following lemma has been proved in [8], Lemma 2.6.

LEMMA 2.1. Let $H<\frac{1}{2}$ and let $n \geq 2$ be an integer. Then there exists a constant $C$ not depending on $T$ such that:

(a) For any $t \in[0, T]$,

$$
\sum_{j=0}^{\lfloor n T\rfloor-1}\left|\left\langle\partial_{\frac{j}{n}}, \varepsilon_{t}\right\rangle_{\mathfrak{H}}\right| \leq C\lfloor n T\rfloor^{2 H} n^{-2 H} .
$$

(b) For any integers $r \geq 1$ and $0 \leq i \leq\lfloor n T\rfloor-1$,

$$
\sum_{j=0}^{\lfloor n T\rfloor-1}\left|\left\langle\partial_{\frac{j}{n}}, \partial_{\frac{i}{n}}\right\rangle_{\mathfrak{H}}^{r}\right| \leq C n^{-2 r H},
$$

and consequently

$$
\sum_{j, i=0}^{\lfloor n T\rfloor-1}\left|\left\langle\partial_{\frac{j}{n}}, \partial_{\frac{i}{n}}\right\rangle_{\mathfrak{H}}^{r}\right| \leq C\lfloor n T\rfloor n^{-2 r H} .
$$

The next result provides useful estimates when we compare two partitions. Its proof is based on computing telescopic sums.

LEMMA 2.2. We fix two integers $n>m \geq 2$, and for any $j \geq 0$, we define $k:=k(j)=\sup \left\{i \geq 0: \frac{i}{m} \leq \frac{j}{n}\right\}$. The following inequalities hold true for some constant $C_{T}$ depending only on $T$ :

$$
\sum_{j=0}^{\lfloor n T\rfloor-1}\left|\left\langle\partial_{\frac{j}{n}}, \varepsilon_{\frac{k(j)}{m}}\right\rangle_{\mathfrak{H}}\right| \leq C_{T} m^{1-2 H},
$$

$$
\sum_{j=0}^{\lfloor n T\rfloor-1}\left|\left\langle\partial_{\frac{j}{n}}, \widetilde{\varepsilon}_{\frac{j}{n}}-\varepsilon_{\frac{k(j)}{m}}\right\rangle_{\mathfrak{H}}\right| \leq C_{T} m^{1-2 H}
$$

and, for any $0 \leq i \leq\lfloor n T\rfloor-1$,

$$
\sum_{j=0}^{\lfloor n T\rfloor-1}\left|\left\langle\partial_{\frac{j}{n}}, \widetilde{\varepsilon}_{\frac{i}{n}}-\varepsilon_{\frac{k(i)}{m}}\right\rangle_{\mathfrak{H}}\right| \leq C_{T} m^{-2 H} .
$$


ProOF. Let us first show (2.11). We can write

$$
\begin{aligned}
\sum_{j=0}^{\lfloor n T\rfloor-1}\left|\left\langle\partial_{\frac{j}{n}}, \varepsilon_{\frac{k(j)}{m}}\right\rangle_{\mathfrak{H}}\right|= & \sum_{j=0}^{\lfloor n T\rfloor-1}\left|\mathbb{E}\left[\left(B_{\frac{j+1}{n}}^{H}-B_{\frac{j}{n}}^{H}\right) B_{\frac{k(j)}{m}}^{H}\right]\right| \\
= & \frac{1}{2} \sum_{j=0}^{\lfloor n T\rfloor-1} \mid\left(\frac{j+1}{n}\right)^{2 H}-\left(\frac{j}{n}\right)^{2 H} \\
& -\left|\frac{j+1}{n}-\frac{k(j)}{m}\right|^{2 H}+\left|\frac{j}{n}-\frac{k(j)}{m}\right|^{2 H} \mid \\
\leq & \frac{1}{2} n^{-2 H} \sum_{j=0}^{\lfloor n T\rfloor-1}\left[(j+1)^{2 H}-j^{2 H}\right] \\
& +\frac{1}{2} \sum_{j=0}^{\lfloor n T\rfloor-1}\left[\left(\frac{j+1}{n}-\frac{k(j)}{m}\right)^{2 H}-\left(\frac{j}{n}-\frac{k(j)}{m}\right)^{2 H}\right] .
\end{aligned}
$$

The first term is a telescopic sum and it is easy to show that

$$
\frac{1}{2} n^{-2 H} \sum_{j=0}^{\lfloor n T\rfloor-1}\left[(j+1)^{2 H}-j^{2 H}\right]=\frac{1}{2} n^{-2 H}(\lfloor n T\rfloor)^{2 H} \leq C_{T} \leq C_{T} m^{1-2 H} .
$$

For the second term, observe that, for a fixed $k=0, \ldots,\lfloor m T\rfloor+1$, the sum of the terms for which $k(j)=k$ is telescopic and is bounded by a constant times $m^{-2 H}$. Summing over all possible values of $k$, we obtain the desired bound $C_{T} m^{1-2 H}$.

Inequality (2.12) is an immediate consequence of (2.11) and the following easy fact:

$$
\begin{aligned}
\sum_{j=0}^{\lfloor n T\rfloor-1}\left|\left\langle\partial_{\frac{j}{n}}, \widetilde{\varepsilon}_{\frac{j}{n}}\right\rangle_{\mathfrak{H}}\right| & =\frac{1}{2} \sum_{j=0}^{\lfloor n T\rfloor-1}\left|\mathbb{E}\left[\left(B_{\frac{j+1}{n}}^{H}-B_{\frac{j}{n}}^{H}\right)\left(B_{\frac{j+1}{n}}^{H}+B_{\frac{j}{n}}^{H}\right)\right]\right| \\
& =\frac{n^{-2 H}}{2} \sum_{j=0}^{\lfloor n T\rfloor-1}\left[(j+1)^{2 H}-j^{2 H}\right] \\
& =\frac{1}{2} n^{-2 H}(\lfloor n T\rfloor)^{2 H} \leq C_{T} \leq C_{T} m^{1-2 H} .
\end{aligned}
$$

Let us now proceed with the proof of (2.13). We can write

$$
\begin{aligned}
& \sum_{j=0}^{\lfloor n T\rfloor-1}\left|\left\langle\partial_{\frac{j}{n}}, \widetilde{\varepsilon}_{\frac{i}{n}}-\varepsilon_{\frac{k(i)}{m}}\right\rangle_{\mathfrak{H}}\right| \\
& \quad=\frac{1}{2} \sum_{j=0}^{\lfloor n T\rfloor-1}\left|\mathbb{E}\left[\left(B_{\frac{j+1}{n}}^{H}-B_{\frac{j}{n}}^{H}\right)\left(B_{\frac{i}{n}}^{H}+B_{\frac{i+1}{n}}^{H}-2 B_{\frac{k(i)}{m}}^{H}\right)\right]\right|
\end{aligned}
$$




$$
\begin{aligned}
\leq & \frac{1}{2} \sum_{j=0}^{\lfloor n T\rfloor-1}\left|\mathbb{E}\left[\left(B_{\frac{j+1}{n}}^{H}-B_{\frac{j}{n}}^{H}\right)\left(B_{\frac{i}{n}}^{H}-B_{\frac{k(i)}{m}}^{H}\right)\right]\right| \\
& +\frac{1}{2} \sum_{j=0}^{\lfloor n T\rfloor-1}\left|\mathbb{E}\left[\left(B_{\frac{j+1}{n}}^{H}-B_{\frac{j}{n}}^{H}\right)\left(B_{\frac{i+1}{n}}^{H}-B_{\frac{k(i)}{m}}^{H}\right)\right]\right| \\
= & : A_{1}+A_{2} .
\end{aligned}
$$

Let us first consider the term $A_{1}$. The main idea to estimate this term is to use the fact that the covariance between the increments $B_{\frac{j+1}{n}}^{H}-B_{\frac{j}{n}}^{H}$ and $B_{\frac{i}{n}}^{H}-B_{\frac{k(i)}{m}}^{H}$ is nonpositive if $j \geq i$ or $j \leq j_{0}$, for some index $j_{0}$ depending on $i$. Then the sums with $j \geq i$ or $j \leq j_{0}$ are telescopic and can be easily estimated. Finally, it suffices to consider the remaining summands. Proceeding in this way, we write

$$
A_{1}=\frac{1}{2} \sum_{j=0}^{\lfloor n T\rfloor-1}\left|H_{j}\right|
$$

where

$$
H_{j}=\left|\frac{i}{n}-\frac{j}{n}\right|^{2 H}-\left|\frac{i}{n}-\frac{j+1}{n}\right|^{2 H}+\left|\frac{k(i)}{m}-\frac{j+1}{n}\right|^{2 H}-\left|\frac{k(i)}{m}-\frac{j}{n}\right|^{2 H} .
$$

Taking into account that $\frac{k(i)}{m} \leq \frac{i}{n}$, it follows that, for $j \geq i$,

$$
\begin{aligned}
H_{j}= & \left(\frac{j}{n}-\frac{i}{n}\right)^{2 H}-\left(\frac{j+1}{n}-\frac{i}{n}\right)^{2 H} \\
& +\left(\frac{j+1}{n}-\frac{k(i)}{m}\right)^{2 H}-\left(\frac{j}{n}-\frac{k(i)}{m}\right)^{2 H} \\
= & 2 H \int_{0}^{\frac{1}{n}}\left[\left(\frac{j}{n}+x-\frac{k(i)}{m}\right)^{2 H-1}-\left(\frac{j}{n}+x-\frac{i}{n}\right)^{2 H-1}\right] d x \\
\leq & 0 .
\end{aligned}
$$

On the other hand, if $j_{0}$ is the largest integer $j \geq 0$ such that $\frac{j+1}{n} \leq \frac{k(i)}{m}$, then, for $j \leq j_{0}$,

$$
\begin{aligned}
H_{j}= & \left(\frac{i}{n}-\frac{j}{n}\right)^{2 H}-\left(\frac{i}{n}-\frac{j+1}{n}\right)^{2 H} \\
& +\left(\frac{k(i)}{m}-\frac{j+1}{n}\right)^{2 H}-\left(\frac{k(i)}{m}-\frac{j}{n}\right)^{2 H} \\
= & -2 H \int_{0}^{\frac{1}{n}}\left[\left(\frac{k(i)}{m}-\frac{j}{n}-x\right)^{2 H-1}-\left(\frac{i}{n}-\frac{j}{n}-x\right)^{2 H-1}\right] d x \\
\leq & 0 .
\end{aligned}
$$


Consider the decomposition

$$
\begin{aligned}
A_{1} & =\frac{1}{2}\left(\sum_{j=0}^{j_{0}}\left|H_{j}\right|+\sum_{j=j_{0}+1}^{i-1}\left|H_{j}\right|+\sum_{j=i}^{\lfloor n T\rfloor-1}\left|H_{j}\right|\right) \\
& =\frac{1}{2}\left(A_{11}+A_{12}+A_{13}\right) .
\end{aligned}
$$

For the terms $A_{11}$ and $A_{13}$, we obtain, respectively,

$$
\begin{aligned}
A_{11} & =\sum_{j=0}^{j_{0}}\left(-H_{j}\right) \\
& =\left(\frac{i}{n}-\frac{j_{0}+1}{n}\right)^{2 H}-\left(\frac{k(i)}{m}-\frac{j_{0}+1}{n}\right)^{2 H}-\left(\frac{i}{n}\right)^{2 H}+\left(\frac{k(i)}{m}\right)^{2 H} \\
& \leq 2\left(\frac{i}{n}-\frac{k(i)}{m}\right)^{2 H} \\
& \leq C m^{-2 H}
\end{aligned}
$$

and

$$
\begin{aligned}
A_{13} & =\sum_{j=i}^{\lfloor n T\rfloor-1}\left(-H_{j}\right) \\
& =\left(\frac{\lfloor n T\rfloor}{n}-\frac{i}{n}\right)^{2 H}-\left(\frac{\lfloor n T\rfloor}{n}-\frac{k(i)}{m}\right)^{2 H}+\left(\frac{i}{n}-\frac{k(i)}{m}\right)^{2 H} \\
& \leq\left(\frac{i}{n}-\frac{k(i)}{m}\right)^{2 H} \\
& \leq m^{-2 H} .
\end{aligned}
$$

Finally, for the term $A_{12}$, we have

$$
\begin{aligned}
A_{12} \leq & \sum_{j=j_{0}+1}^{i-1}\left|\left(\frac{i}{n}-\frac{j}{n}\right)^{2 H}-\left(\frac{i}{n}-\frac{j+1}{n}\right)^{2 H}\right| \\
& +\sum_{j=j_{0}+1}^{i-1}\left|\left(\frac{j+1}{n}-\frac{k(i)}{m}\right)^{2 H}-\left(\frac{j}{n}-\frac{k(i)}{m}\right)^{2 H}\right| \\
= & : A_{121}+A_{122} .
\end{aligned}
$$

The term $A_{121}$ is a telescopic sum which produces a contribution of the form

$$
\left(\frac{i-j_{0}-1}{n}\right)^{2 H} \leq C_{T} m^{-2 H}
$$


and the term $A_{122}$ can be bounded as follows:

$$
\begin{aligned}
A_{122} \leq & \left|\frac{j_{0}+2}{n}-\frac{k(i)}{m}\right|^{2 H}+\left|\frac{j_{0}+1}{n}-\frac{k(i)}{m}\right|^{2 H} \\
& +\sum_{j=j_{0}+2}^{i-1}\left[\left(\frac{j+1}{n}-\frac{k(i)}{m}\right)^{2 H}-\left(\frac{j}{n}-\frac{k(i)}{m}\right)^{2 H}\right] \\
\leq & C_{T} m^{-2 H}+\left(\frac{i}{n}-\frac{k(i)}{m}\right)^{2 H}-\left(\frac{j_{0}+2}{n}-\frac{k(i)}{m}\right)^{2 H} \\
\leq & C_{T} m^{-2 H} .
\end{aligned}
$$

The term $A_{2}$ can be treated in a similar way. This completes the proof.

We will use the following lemma.

LEMMA 2.3. For any odd integer $r \geq 1$, we have

$$
\left(\Delta_{j}^{n} B^{H}\right)^{r}=\sum_{u=0}^{\left\lfloor\frac{r}{2}\right\rfloor} C_{r, u} n^{-2 u H} I_{r-2 u}\left(\partial_{\frac{j}{n}}^{\otimes r-2 u}\right),
$$

where $C_{r, u}$ are some integers.

ProOF. By (2.7), we have $\left\|\Delta_{j}^{n} B^{H}\right\|_{L^{2}(\Omega)}=n^{-H}$. For any integer $q \geq 1$, we recall [see (2.2)] that $H_{q}(x)$ denotes the Hermite polynomial of degree $q$. Using an inductive argument coming from the relation $H_{q+1}(x)=x H_{q}(x)-q H_{q-1}(x)$, it follows that

$$
x^{r}=\sum_{u=0}^{\left\lfloor\frac{r}{2}\right\rfloor} C_{r, u} H_{r-2 u}(x),
$$

where $C_{r, u}$ is an integer. Applying (2.3) to $h=n^{H} \partial_{\frac{j}{n}}$, that is, $X(h)=\Delta_{j}^{n} B^{H} /$ $\left\|\Delta_{j}^{n} B^{H}\right\|_{L^{2}(\Omega)}=n^{H} \Delta_{j}^{n} B^{H}$, we can write

$$
H_{r}\left(n^{H} \Delta_{j}^{n} B^{H}\right)=I_{r}\left(n^{r H} \partial_{\frac{j}{n}}^{\otimes r}\right) .
$$

Substituting (2.15) into (2.14) yields

$$
n^{r H}\left(\Delta_{j}^{n} B^{H}\right)^{r}=\sum_{u=0}^{\left\lfloor\frac{r}{2}\right\rfloor} C_{r, u} I_{r-2 u}\left(n^{(r-2 u) H} \partial_{\frac{j}{n}}^{\otimes r-2 u}\right),
$$

which implies the desired result. 
3. Proof of Theorem 1.1. We recall that $\ell=\ell(v)$ is defined by (1.2). The first ingredient of the proof is the following expansion, established in [5], based on Taylor's formula and the properties of the measure $v$ :

$$
\begin{aligned}
f(b)= & f(a)+(b-a) \int_{0}^{1} f^{\prime}(a+\alpha(b-a)) v(d \alpha) \\
& +\sum_{h=\ell}^{2 \ell} k_{\nu, h} f^{(2 h+1)}\left(\frac{a+b}{2}\right)(b-a)^{2 h+1} \\
& +(b-a)^{4 \ell+2} C(a, b),
\end{aligned}
$$

where $a, b \in \mathbb{R}$ and $C(a, b)$ is a continuous function such that $C(a, a)=0$. The constants $k_{v, h}$ are given by

$$
k_{v, h}=\frac{1}{(2 h) !}\left[\frac{1}{(2 h+1) 4^{h}}-\int_{0}^{1}\left(\alpha-\frac{1}{2}\right)^{2 h} v(d \alpha)\right] .
$$

Applying equality (3.1) to $a=B_{\frac{j}{n}}^{H}$ and $b=B_{\frac{j+1}{n}}^{H}$ and using the notation $\widetilde{B}_{\frac{j}{n}}^{H}=$ $\frac{1}{2}\left(B_{\frac{j}{n}}^{H}+B_{\frac{j+1}{n}}^{H}\right)$ and $\Delta_{j}^{n} B^{H}=B_{\frac{j+1}{n}}^{H}-B_{\frac{j}{n}}^{H}$ yields

$$
\begin{aligned}
f\left(B_{\lfloor n t\rfloor}^{H}\right)-f(0)= & \sum_{j=0}^{\lfloor n t\rfloor-1} \Delta_{j}^{n} B^{H} \int_{0}^{1} f^{\prime}\left(B_{\frac{j}{n}}^{H}+\alpha \Delta_{j}^{n} B^{H}\right) v(d \alpha) \\
& +\sum_{h=\ell}^{2 \ell} \sum_{j=0}^{\lfloor n t\rfloor-1} k_{\nu, h} f^{(2 h+1)}\left(\widetilde{B}_{\frac{j}{n}}^{H}\right)\left(\Delta_{j}^{n} B^{H}\right)^{2 h+1} \\
& +\sum_{j=0}^{\lfloor n\rfloor\rfloor-1} C\left(B_{\frac{j}{n}}^{H}, B_{\frac{j+1}{n}}^{H}\right)\left(\Delta_{j}^{n} B^{H}\right)^{4 \ell+2},
\end{aligned}
$$

which can be written as

$$
f\left(B_{\lfloor n t\rfloor}^{H}\right)-f(0)=S_{n}^{\nu}\left(f^{\prime}, t\right)+\sum_{h=\ell}^{2 \ell} \Phi_{n}^{h}(t)+R_{n}(t),
$$

where, for each $h=\ell, \ldots, 2 \ell$,

$$
\Phi_{n}^{h}(t)=\sum_{j=0}^{\lfloor n t\rfloor-1} k_{v, h} f^{(2 h+1)}\left(\widetilde{B}_{\frac{j}{n}}^{H}\right)\left(\Delta_{j}^{n} B^{H}\right)^{2 h+1}
$$

and

$$
R_{n}(t)=\sum_{j=0}^{\lfloor n t\rfloor-1} C\left(B_{\frac{j}{n}}^{H}, B_{\frac{j+1}{n}}^{H}\right)\left(\Delta_{j}^{n} B^{H}\right)^{4 \ell+2} .
$$


Let us consider the convergence of each term in the decomposition (3.3). First, we will show that the term $R_{n}(t)$ converges to zero in probability, uniformly in compact sets. In fact, for any $T>0, K, \epsilon>0$, we can write

$$
\begin{aligned}
P\left(\sup _{0 \leq t \leq T}\left|R_{n}(t)\right|>\epsilon\right) \\
\leq P\left(\sup _{\substack{s, t \in[0, T] \\
|t-s| \leq \frac{1}{n}}}\left|C\left(B_{s}^{H}, B_{t}^{H}\right)\right|>\frac{1}{K}\right) \\
\quad+P\left(\sum_{j=0}^{\lfloor n T\rfloor-1}\left(\Delta_{j}^{n} B^{H}\right)^{4 \ell+2}>K \epsilon\right) .
\end{aligned}
$$

Taking into account that $H=\frac{1}{4 \ell+2}$ and using (2.7), we can write, with $\mu_{k}$ denoting the $k$ th moment of the standard Gaussian distribution,

$$
P\left(\sum_{j=0}^{\lfloor n T\rfloor-1}\left(\Delta_{j}^{n} B^{H}\right)^{4 \ell+2}>K \epsilon\right) \leq \frac{\mu_{4 \ell+2}}{K \epsilon} \frac{\lfloor n T\rfloor}{n} \leq \frac{T \mu_{4 \ell+2}}{K \epsilon} .
$$

From (3.5) and (3.6), letting first $n \rightarrow \infty$ and then $K \rightarrow \infty$ it follows that for any $\epsilon>0$ and $T>0$,

$$
\lim _{n \rightarrow \infty} P\left(\sup _{0 \leq t \leq T}\left|R_{n}(t)\right|>\epsilon\right)=0 .
$$

On the other hand, by Lemma A.1, the terms $\Phi_{n}^{h}$ with $h=\ell+1, \ldots, 2 \ell$ converge to zero in the topology of $D([0, \infty))$ and do not contribute to the limit. As a consequence, the proof of Theorem 1.1 follows from the next proposition.

PROPOSITION 3.1. Under the assumptions of Theorem 1.1, one has

$$
\Phi_{n}^{\ell}(t)=\sum_{j=0}^{\lfloor n t\rfloor-1} f^{(2 \ell+1)}\left(\widetilde{B}_{\frac{j}{n}}^{H}\right)\left(\Delta_{j}^{n} B^{H}\right)^{2 \ell+1} \underset{\mathrm{n} \rightarrow \infty}{\stackrel{\mathcal{L}}{\longrightarrow}} \sigma_{\ell} \int_{0}^{t} f^{(2 \ell+1)}\left(B_{s}^{H}\right) d W_{s}
$$

where $W=\left\{W_{t}, t \geq 0\right\}$ is a Brownian motion independent of $B^{H}, \sigma_{\ell}$ is the constant defined in (1.4), and the convergence holds in the topology of the Skorohod space $D([0, \infty))$.

PROOF. In order to show Proposition 3.1, we will first prove that the sequence of processes $\left\{\Phi_{n}^{\ell}(t), t \geq 0\right\}$ is tight in $D([0, \infty))$, and then that their finite dimensional distributions converge to those of

$$
\left\{\sigma_{\ell} \int_{0}^{t} f^{(2 \ell+1)}\left(B_{s}^{H}\right) d W_{s}, t \geq 0\right\} .
$$


Notice that the tightness of the sequence $\Phi_{n}^{\ell}$ is a consequence of Lemma A.1. Indeed, this lemma implies that for any $0 \leq s<t \leq T$, there exist a constant $C_{T}$ depending on $T$, such that

$$
\mathbb{E}\left[\left|\Phi_{n}^{\ell}(t)-\Phi_{n}^{\ell}(s)\right|^{4}\right] \leq C_{T} \sum_{N=2}^{4}\left(\frac{\lfloor n t\rfloor-\lfloor n s\rfloor}{n}\right)^{N} .
$$

It remains to show the convergence of the finite-dimensional distributions. Fix a finite set of points $0 \leq t_{1}<\cdots \leq t_{d} \leq T$. We want to show the following convergence in law, as $n$ tends to infinity:

$$
\left(\Phi_{n}^{\ell}\left(t_{1}\right), \ldots, \Phi_{n}^{\ell}\left(t_{d}\right)\right) \underset{\mathrm{n} \rightarrow \infty}{\stackrel{\mathcal{L}}{\longrightarrow}}\left(Y_{1}, \ldots, Y_{d}\right),
$$

where

$$
Y_{i}=\sigma_{\ell} \int_{0}^{t_{i}} f^{(2 \ell+1)}\left(B_{s}^{H}\right) d W_{s}, \quad i=1, \ldots, d,
$$

$W=\left\{W_{t}, t \geq 0\right\}$ is a Brownian motion independent of $B^{H}$, and $\sigma_{\ell}$ is the constant defined in (1.4).

Taking into account the convergence (1.6), the main ingredient in the proof of the convergence (3.8) is the methodology based on the small blocks/big blocks (see, for instance, [4]). This method consists in considering two integers $2 \leq m<n$ and let first $n$ tend to infinity and later $m$ tend to infinity. For any $k \geq 0$, we define the set

$$
I_{k}=\left\{j \in\left\{0, \ldots,\left\lfloor n t_{i}\right\rfloor-1\right\}: \frac{k}{m} \leq \frac{j}{n}<\frac{k+1}{m}\right\} .
$$

The basic ingredient in this approach is the decomposition

$$
\begin{aligned}
\Phi_{n}^{\ell}\left(t_{i}\right)= & \sum_{k=0}^{\left\lfloor m t_{i}\right\rfloor} \sum_{j \in I_{k}} f^{(2 \ell+1)}\left(B_{\frac{k}{m}}^{H}\right)\left(\Delta_{j}^{n} B^{H}\right)^{2 \ell+1} \\
& +\sum_{k=0}^{\left\lfloor m t_{i}\right\rfloor} \sum_{j \in I_{k}}\left[f^{(2 \ell+1)}\left(\widetilde{B}_{\frac{j}{n}}^{H}\right)-f^{(2 \ell+1)}\left(B_{\frac{k}{m}}^{H}\right)\right]\left(\Delta_{j}^{n} B^{H}\right)^{2 \ell+1} \\
=: & A_{n, m}^{(1, i)}+A_{n, m}^{(2, i) .}
\end{aligned}
$$

From Lemma A.2 with $r=2 \ell+1$ and $\phi=f^{(2 \ell+1)}$, we can write, for any $q>2$,

$$
\begin{aligned}
& \mathbb{E}\left[\left(A_{n, m}^{(2, i)}\right)^{2}\right] \\
& \quad \leq C_{T} \sup _{0 \leq w \leq 3(2 \ell+1)} \sup _{0 \leq j \leq\lfloor n T\rfloor-1}\left\|f^{(w)}\left(\widetilde{B}_{\frac{j}{n}}^{H}\right)-f^{(w)}\left(B_{\frac{k(j)}{m}}^{H}\right)\right\|_{L^{q}(\Omega)}^{2} \\
& \quad+C_{T} \sup _{0 \leq w \leq 3(2 \ell+1)} \sup _{0 \leq j \leq\lfloor n T\rfloor-1}\left\|f^{(w)}\left(\widetilde{B}_{\frac{j}{n}}^{H}\right)\right\|_{L^{2}(\Omega)}^{2}\left(m^{-2 H}+n^{2 H-1} m^{2-4 H}\right)
\end{aligned}
$$




$$
\begin{aligned}
& +C_{T} \sup _{0 \leq w \leq 3(2 \ell+1)} \sup _{0 \leq i, j \leq\lfloor n T\rfloor-1}\left\|f^{(w)}\left(\widetilde{B}_{\frac{i}{n}}^{H}\right)\right\|_{L^{2}(\Omega)} \\
& \times\left\|f^{(w)}\left(\widetilde{B}_{\frac{j}{n}}^{H}\right)-f^{(w)}\left(B_{\frac{k(j)}{m}}^{H}\right)\right\|_{L^{2}(\Omega)}\left(1+n^{2 H-1} m^{2-4 H}\right) \\
\leq & C_{T}\left[m^{-2 H}+n^{2 H-1} m^{2-4 H}\right. \\
& \left.\times\left(1+\sup _{0 \leq w \leq 3(2 \ell+1)} \sup _{\substack{s, t \in[0, T] \\
|t-s| \leq \frac{1}{m}}}\left\|f^{(w)}\left(\frac{B_{s}^{H}+B_{s+\frac{1}{n}}^{H}}{2}\right)-f^{(w)}\left(B_{t}^{H}\right)\right\|_{L^{q}(\Omega)}^{2}\right)\right],
\end{aligned}
$$

where $k:=k(j)=\sup \left\{i \geq 0: \frac{i}{m} \leq \frac{j}{n}\right\}$. This implies

$$
\begin{aligned}
& \limsup _{n \rightarrow \infty} \mathbb{E}\left[\left(A_{n, m}^{(2, i)}\right)^{2}\right] \\
& \quad \leq C_{T}\left(m^{-2 H}+\sup _{0 \leq w \leq 3(2 \ell+1)} \sup _{\substack{s, t \in[0, T] \\
|t-s| \leq \frac{1}{m}}}\left\|f^{(w)}\left(B_{s}^{H}\right)-f^{(w)}\left(B_{t}^{H}\right)\right\|_{L^{2}(\Omega)}^{2}\right),
\end{aligned}
$$

which converges to zero as $m$ tends to infinity.

On the other hand, from (1.6) we deduce that the vector $\left(A_{n, m}^{(1,1)}, \ldots, A_{n, m}^{(1, d)}\right)$ converges in law, as $n$ tends to infinity, to the vector with components

$$
\sigma_{\ell} \sum_{k=0}^{\left\lfloor m t_{i}\right\rfloor} f^{(2 \ell+1)}\left(B_{\frac{k}{m}}^{H}\right)\left(W_{\frac{k+1}{m}}-W_{\frac{k}{m}}\right)
$$

$i=1, \ldots, d$, where $W$ is a Brownian motion independent of $B^{H}$. Each of these components converges in $L^{2}(\Omega)$ to the stochastic integral $\sigma_{\ell} \int_{0}^{t_{i}} f^{(2 \ell+1)}\left(B_{s}^{H}\right) d W_{s}$, as $m$ tends to infinity. This completes the proof of the theorem.

\section{APPENDIX}

This section is devoted to state and prove a couple of technical lemmas. The first lemma is the basic ingredient to show that the sequence of processes $\Phi_{n}^{\ell}$ is tight and the processes $\Phi_{n}^{h}$ for $h=\ell+1, \ldots, 2 \ell$ converge to zero in $D([0, \infty))$. For this, we need to estimate the fourth moment of the increments of the processes $\Phi_{n}^{h}$.

LEMMA A.1. Consider the processes $\Phi_{n}^{h}, h=\ell, \ldots, 2 \ell$ defined in (3.4). Then, for any $0 \leq s<t \leq T$, we have

$$
\mathbb{E}\left[\left|\Phi_{n}^{h}(t)-\Phi_{n}^{h}(s)\right|^{4}\right] \leq C_{T} \sum_{N=2}^{4}(\lfloor n t\rfloor-\lfloor n s\rfloor)^{N} n^{-2 N H(2 h+1)},
$$

where the constant $C_{T}$ depends only on $T$. 
ProOF. For any $0 \leq s<t \leq T$, we can write

$$
\mathbb{E}\left[\left|\Phi_{n}(t)-\Phi_{n}(s)\right|^{4}\right]=\sum_{j_{1}, j_{2}, j_{3}, j_{4}=\lfloor n s\rfloor}^{\lfloor n t\rfloor-1} \mathbb{E}\left[\prod_{i=1}^{4}\left(f^{(2 h+1)}\left(\widetilde{B}_{\frac{j_{i}}{n}}^{H}\right)\left(\Delta_{j_{i}}^{n} B^{H}\right)^{2 h+1}\right)\right] .
$$

By Lemma 2.3, we obtain

$$
\left(\Delta_{j_{i}}^{n} B^{H}\right)^{2 h+1}=\sum_{u=0}^{h} C_{2 h+1, u} n^{-2 u H} I_{2 h+1-2 u}\left(\partial_{\frac{j_{i}}{n}}^{\otimes(2 h+1-2 u)}\right),
$$

which leads to

$$
\prod_{i=1}^{4}\left(\Delta_{j_{i}}^{n} B^{H}\right)^{2 h+1}=\sum_{u_{1}, u_{2}, u_{3}, u_{4}=0}^{h} C_{h, \mathbf{u}} n^{-2|\mathbf{u}| H} \prod_{i=1}^{4}\left(I_{2 h+1-2 u_{i}}\left(\partial_{\frac{j_{i}}{n}}^{\otimes\left(2 h+1-2 u_{i}\right)}\right)\right),
$$

where $C_{h, \mathbf{u}}$ is a constant depending on $h$ and the vector $\mathbf{u}=\left(u_{1}, u_{2}, u_{3}, u_{4}\right)$ and we use the notation $|\mathbf{u}|=u_{1}+u_{2}+u_{3}+u_{4}$. To simplify the notation, we write $2 h+1-u_{i}=v_{i}$ for $i=1,2,3,4$. The product formula for multiple stochastic integrals (2.4) allows us to write

$$
\begin{aligned}
& \prod_{i=1}^{4}\left(I_{v_{i}}\left(\partial_{\frac{j_{i}}{n}}^{\otimes v_{i}}\right)\right) \\
& =\sum_{\alpha \in \Lambda} C_{\alpha} \prod_{1 \leq i<k \leq 4}\left\langle\partial_{\frac{j_{i}}{n}}, \partial_{\frac{j_{k}}{n}}\right\rangle_{\mathcal{H}}^{\alpha_{i k}} \\
& \times I_{|\mathbf{v}|-2|\alpha|}\left(\partial_{\frac{j_{1}}{n}}^{\otimes^{v_{1}-\alpha_{12}-\alpha_{13}-\alpha_{14}}} \otimes \partial_{\frac{j_{2}}{n}}^{\otimes v_{2}-\alpha_{12}-\alpha_{23}-\alpha_{24}}\right.
\end{aligned}
$$

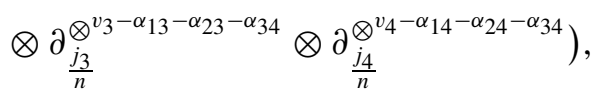

where $|\mathbf{v}|=v_{1}+v_{2}+v_{3}+v_{4}=8 h+4-|\mathbf{u}|, \Lambda$ is the set of all multiindices $\alpha=\left(\alpha_{12}, \alpha_{13}, \alpha_{14}, \alpha_{23}, \alpha_{24}, \alpha_{34}\right)$ with $\alpha_{i k} \geq 0$, such that

$$
\begin{aligned}
& \alpha_{12}+\alpha_{13}+\alpha_{14} \leq v_{1}, \\
& \alpha_{12}+\alpha_{23}+\alpha_{24} \leq v_{2}, \\
& \alpha_{13}+\alpha_{23}+\alpha_{34} \leq v_{3}, \\
& \alpha_{14}+\alpha_{24}+\alpha_{34} \leq v_{4} .
\end{aligned}
$$

For any $\mathbf{j}=\left(j_{1}, j_{2}, j_{3}, j_{4}\right),\lfloor n s\rfloor \leq j_{i} \leq\lfloor n t\rfloor-1$, we set

$$
Y_{\mathbf{j}}=\prod_{i=1}^{4} f^{(2 h+1)}\left(\widetilde{B}_{\frac{j_{i}}{n}}^{H}\right)
$$


and

$$
\begin{aligned}
h_{\mathbf{j}, \boldsymbol{\alpha}, \mathbf{v}}= & \partial_{\frac{j_{1}}{n}}^{\otimes^{v_{1}-\alpha_{12}-\alpha_{13}-\alpha_{14}}} \otimes \partial_{\frac{j_{2}}{n}}^{\otimes^{v_{2}-\alpha_{12}-\alpha_{23}-\alpha_{24}}} \\
& \otimes \partial_{\frac{j_{3}}{n}}^{\otimes^{v_{3}-\alpha_{13}-\alpha_{23}-\alpha_{34}}} \otimes \partial_{\frac{j_{4}}{n}}^{\otimes^{v_{4}-\alpha_{14}-\alpha_{24}-\alpha_{34}} .}
\end{aligned}
$$

Applying the duality formula (2.6), we obtain

$$
\mathbb{E}\left[Y_{\mathbf{j}} I_{|\mathbf{v}|-2|\alpha|}\left(h_{\mathbf{j}, \boldsymbol{\alpha}, \mathbf{v}}\right)\right]=\mathbb{E}\left[\left\langle D^{|\mathbf{v}|-2|\alpha|} Y_{\mathbf{j}}, h_{\mathbf{j}, \boldsymbol{\alpha}, \mathbf{v}}\right\rangle_{\mathcal{H} \otimes|\mathbf{v}|-2|\alpha|}\right] .
$$

Therefore, we have shown the following formula:

$$
\begin{aligned}
& \mathbb{E}\left[\left|\Phi_{n}^{h}(t)-\Phi_{n}^{h}(s)\right|^{4}\right]=\sum_{\mathbf{j}} \sum_{\mathbf{u}} C_{h, \mathbf{u}} n^{-2|\mathbf{u}| H} \sum_{\alpha \in \Lambda} C_{\alpha} \\
& \times\left(\prod_{1 \leq i<k \leq 4}\left\langle\partial_{\frac{j_{i}}{n}}, \partial_{\frac{j_{k}}{n}}\right\rangle_{\mathcal{H}}^{\alpha_{i k}}\right) \mathbb{E}\left[\left\langle D^{|\mathbf{v}|-2|\alpha|} Y_{\mathbf{j}}, h_{\mathbf{j}, \boldsymbol{\alpha}, \mathbf{v}}\right\rangle_{\mathcal{H}}^{\otimes|\mathbf{v}|-2|\alpha|}\right],
\end{aligned}
$$

where the components of $\mathbf{j}$ satisfy $\lfloor n s\rfloor \leq j_{i} \leq\lfloor n t\rfloor-1$ and $0 \leq u_{i} \leq h$. Finally,

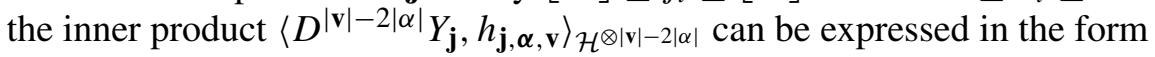

$$
\sum_{\beta \in \Gamma} \Phi_{\beta} \prod_{1 \leq i, k \leq 4}\left\langle\partial_{\frac{j_{i}}{n}}, \widetilde{\varepsilon}_{\frac{j_{k}}{n}}\right\rangle_{\mathcal{H}}^{\beta_{i k}},
$$

where $\beta=\left(\beta_{i k}\right)_{1 \leq i, k \leq 4}$ is a matrix with nonnegative entries such that

$$
\begin{aligned}
& \sum_{k=1}^{4} \beta_{1 k}=v_{1}-\alpha_{12}-\alpha_{13}-\alpha_{14}, \\
& \sum_{k=1}^{4} \beta_{2 k}=v_{2}-\alpha_{12}-\alpha_{23}-\alpha_{24}, \\
& \sum_{k=1}^{4} \beta_{3 k}=v_{3}-\alpha_{13}-\alpha_{23}-\alpha_{34}, \\
& \sum_{k=1}^{4} \beta_{4 k}=v_{4}-\alpha_{14}-\alpha_{24}-\alpha_{34} .
\end{aligned}
$$

Notice that $|\beta|=\sum_{i, k=1}^{4} \beta_{i k}=|\mathbf{v}|-2|\alpha|$. Moreover, the random variables $\Phi_{\beta}$ are linear combinations of products of the form $\prod_{i=1}^{4} f^{\left(w_{i}\right)}\left(B_{\tilde{\varepsilon}_{\frac{j_{i}}{n}}^{H}}\right)$, with $2 h+1 \leq w_{i} \leq$ $2 h+1+|\mathbf{v}|-2|\alpha|$. This leads to the following estimate:

$$
\begin{aligned}
& \mathbb{E}\left[\left|\Phi_{n}^{h}(t)-\Phi_{n}^{h}(s)\right|^{4}\right] \\
& \quad \leq C_{T} \sum_{\mathbf{j}} \sum_{\mathbf{u}} n^{-2|\mathbf{u}| H} \sum_{\alpha \in \Lambda} \sum_{\beta \in \Gamma} \prod_{1 \leq i<k \leq 4}\left|\left\langle\partial_{\frac{j_{i}}{n}}, \partial_{\frac{j_{k}}{n}}\right\rangle_{\mathcal{H}}^{\alpha_{i k}}\right| \prod_{1 \leq i, k \leq 4}\left|\left\langle\partial_{\frac{j_{i}}{n}}, \widetilde{\varepsilon}_{\frac{j_{k}}{n}}\right\rangle_{\mathcal{H}}^{\beta_{i k}}\right| .
\end{aligned}
$$


Consider the decomposition of the above sum as follows:

$$
\mathbb{E}\left[\left|\Phi_{n}(t)-\Phi_{n}(s)\right|^{4}\right] \leq C_{T}\left(A_{n}^{(1)}+A_{n}^{(2)}+A_{n}^{(3)}\right),
$$

where $A_{n}^{(1)}$ contains all the terms such that at least two components of $\alpha$ are nonzero, $A_{n}^{(2)}$ contains all the terms such that one component of $\alpha$ is nonzero and the others vanish and $A_{n}^{(3)}$ contains all the terms such that all the components of $\alpha$ are zero.

Step 1 . Let us first estimate $A_{n}^{(1)}$. Without any loss of generality, we can assume that $\alpha_{12} \geq 1$ and $\alpha_{13} \geq 1$. From (2.9) with $r=1$, we obtain

$$
\sum_{j_{1}=\lfloor n s\rfloor}^{\lfloor n t\rfloor-1}\left|\left\langle\partial_{\frac{j_{1}}{n}}, \partial_{\frac{j_{2}}{n}}\right\rangle_{\mathcal{H}}\right| \leq C n^{-2 H}
$$

and

$$
\sum_{j_{3}=\lfloor n s\rfloor}^{\lfloor n t\rfloor-1}\left|\left\langle\partial_{\frac{j_{1}}{n}}, \partial_{\frac{j_{3}}{n}}\right\rangle_{\mathcal{H}}\right| \leq C n^{-2 H} .
$$

We estimate each of the remaining factors by $n^{-2 H}$. In this way, we obtain a bound of the form

$$
A_{n}^{(1)} \leq C(\lfloor n t\rfloor-\lfloor n s\rfloor)^{2} n^{-2 H(|\mathbf{u}|+|\alpha|+|\beta|)} .
$$

Taking into account that $|\alpha| \leq \frac{1}{2}|\mathbf{v}|$, we can write

$$
\begin{aligned}
|\mathbf{u}|+|\alpha|+|\beta| & =|\mathbf{u}|+|\alpha|+|\mathbf{v}|-2|\alpha| \\
& =|\mathbf{u}|+|\mathbf{v}|-|\alpha| \\
& \geq|\mathbf{u}|+\frac{|\mathbf{v}|}{2} \\
& =4 h+2-\frac{|\mathbf{u}|}{2} \\
& \geq 4 h+2,
\end{aligned}
$$

and, as a consequence,

$$
A_{n}^{(1)} \leq C(\lfloor n t\rfloor-\lfloor n s\rfloor)^{2} n^{-4 H(2 h+1)} .
$$

Step 2. For the term $A_{n}^{(2)}$, we can assume that $\alpha_{12} \geq 1$ and all the other components of $\alpha$ vanish. In this case, we still have the inequality (A.1). Then we estimate each of the remaining factors by $n^{-2 H}$. In this way, we obtain a bound of the form

$$
A_{n}^{(2)} \leq C(\lfloor n t\rfloor-\lfloor n s\rfloor)^{3} n^{-2 H(|\mathbf{u}|+|\alpha|+|\beta|)} .
$$


Taking into account that $|\alpha|=\alpha_{12} \leq v_{1}=2 h+1-u_{1} \leq 2 h+1$, we can write

$$
\begin{aligned}
|\mathbf{u}|+|\alpha|+|\beta| & =|\mathbf{u}|+|\alpha|+|\mathbf{v}|-2|\alpha| \\
& =|\mathbf{u}|+|\mathbf{v}|-|\alpha| \\
& \geq|\mathbf{u}|+|\mathbf{v}|-2 h-1 \\
& =6 h+3,
\end{aligned}
$$

and, as a consequence, we obtain

$$
A_{n}^{(2)} \leq C(\lfloor n t\rfloor-\lfloor n s\rfloor)^{3} n^{-6 H(2 h+1)} .
$$

Step 3. Estimating all terms by $n^{-2 H}$, we get

$$
A_{n}^{(3)} \leq C(\lfloor n t\rfloor-\lfloor n s\rfloor)^{4} n^{-2 H(|\mathbf{u}|+|\beta|)} .
$$

We have

$$
|\mathbf{u}|+|\beta|=|\mathbf{u}|+|\mathbf{v}|=8 h+4,
$$

and, as a consequence, we obtain

$$
A_{n}^{(3)} \leq C(\lfloor n t\rfloor-\lfloor n s\rfloor)^{4} n^{-8 H(2 h+1)} .
$$

In conclusion, from (A.2), (A.3) and (A.4), we obtain the desired estimate. This completes the proof of the lemma.

The second lemma provides a bound for the residual term in the application of the small blocks/big blocks technique and it is a variation of [8], Lemma 3.2. Its proof is based on the techniques of Malliavin calculus. As before, for two integers $n>m \geq 2$, for any $j \geq 0$ we define $k:=k(j)=\sup \left\{i \geq 0: \frac{i}{m} \leq \frac{j}{n}\right\}$.

LEMMA A.2. Let $r=1,3,5, \ldots$ and $n>m \geq 2$ be two integers. Let $\phi: \mathbb{R} \rightarrow$ $\mathbb{R}$ be a $\mathcal{C}^{2 r}$ function such that $\phi$ and all derivatives up to order $2 r$ have moderate growth, and let $B^{H}=\left\{B_{t}^{H}, t \geq 0\right\}$ be a fBm with Hurst parameter $H<\frac{1}{2}$. Then, for any $q>2$ and any $T>0$,

$$
\sup _{t \in[0, T]} \mathbb{E}\left[\left(\sum_{j=0}^{\lfloor n t\rfloor-1}\left(\phi\left(\widetilde{B}_{\frac{j}{n}}^{H}\right)-\phi\left(B_{\frac{k(j)}{m}}\right)\right)\left(\Delta_{j}^{n} B^{H}\right)^{r}\right)^{2}\right] \leq C_{T} \Gamma_{m, n} n^{1-2 r H},
$$

where $C_{T}$ is a positive constant depending on $q, r, H$ and $T$ and

$$
\begin{aligned}
\Gamma_{m, n}:= & \sup _{0 \leq w \leq 2 r} \sup _{0 \leq j \leq\lfloor n T\rfloor-1}\left\|\phi^{(w)}\left(\widetilde{B}_{\frac{j}{n}}^{H}\right)-\phi^{(w)}\left(B_{\frac{k(j)}{m}}^{H}\right)\right\|_{L^{q}(\Omega)}^{2} \\
& +\sup _{0 \leq w \leq 2 r} \sup _{0 \leq j \leq\lfloor n T\rfloor-1}\left\|\phi^{(w)}\left(\widetilde{B}_{\frac{j}{n}}^{H}\right)\right\|_{L^{2}(\Omega)}^{2}\left(m^{-2 H}+n^{2 H-1} m^{2-4 H}\right) \\
& +\sup _{0 \leq w \leq 2 r} \sup _{0 \leq i, j \leq\lfloor n T\rfloor-1}\left\|\phi^{(w)}\left(\widetilde{B}_{\frac{i}{n}}^{H}\right)\right\|_{L^{2}(\Omega)}\left\|\phi^{(w)}\left(\widetilde{B}_{\frac{j}{n}}^{H}\right)-\phi^{(w)}\left(B_{\frac{k(j)}{n}}^{H}\right)\right\|_{L^{2}(\Omega)} \\
& \times\left(1+n^{2 H-1} m^{2-4 H}\right) .
\end{aligned}
$$


Proof. The proof is based in the methodology used to show Lemma 3.2 in [8]. To simplify notation, let $Y_{j}(\phi):=\phi\left(\widetilde{B}_{\frac{j}{n}}^{H}\right)-\phi\left(B_{\frac{k(j)}{m}}^{H}\right)$, and set

$$
I_{t}:=\mathbb{E}\left[\left(\sum_{j=0}^{\lfloor n t\rfloor-1} Y_{j}(\phi)\left(\Delta_{j}^{n} B^{H}\right)^{r}\right)^{2}\right] .
$$

From Lemma 2.3, we obtain

$$
\begin{aligned}
I_{t} & =\sum_{u, v=0}^{\left\lfloor\frac{r}{2}\right\rfloor} C_{r, u} C_{r, v} n^{-2 H(u+v)} \sum_{i, j=0}^{\lfloor n t\rfloor-1} \mathbb{E}\left[Y_{i}(\phi) Y_{j}(\phi) I_{r-2 u}\left(\partial_{\frac{i}{n}}^{\otimes r-2 u}\right) I_{r-2 v}\left(\partial_{\frac{j}{n}}^{\otimes r-2 v}\right)\right] \\
& \leq C \sum_{u, v=0}^{\left\lfloor\frac{r}{2}\right\rfloor} n^{-2 H(u+v)} \sum_{i, j=0}^{\lfloor n T\rfloor-1}\left|\mathbb{E}\left[Y_{i}(\phi) Y_{j}(\phi) I_{r-2 u}\left(\partial_{\frac{i}{n}}^{\otimes r-2 u}\right) I_{r-2 v}\left(\partial_{\frac{j}{n}}^{\otimes r-2 v}\right)\right]\right| .
\end{aligned}
$$

Then we apply the product formula (2.4) in order to develop the product of two multiple stochastic integrals and we end up with

$$
\begin{aligned}
& I_{t} \leq C \sum_{u, v=0}^{\left\lfloor\frac{r}{2}\right\rfloor} \sum_{z=0}^{(r-2 u) \wedge(r-2 v)} n^{-2 H(u+v)} \sum_{i, j=0}^{\lfloor n T\rfloor-1} \mid \mathbb{E}\left[Y_{i}(\phi) Y_{j}(\phi)\right. \\
& \left.\times I_{2 r-2}(u+v)-2 z\left(\partial_{\frac{i}{n}}^{\otimes r-2 u-z} \widetilde{\otimes} \partial_{\frac{j}{n}}^{\otimes r-2 v-z}\right)\left\langle\partial_{\frac{i}{n}}, \partial_{\frac{j}{n}}\right\rangle_{\mathfrak{H}}^{z}\right] \mid \\
& =C \sum_{u, v=0}^{\left\lfloor\frac{r}{2}\right\rfloor} n^{-2 H(u+v)} \sum_{i, j=0}^{\lfloor n T\rfloor-1}\left|\mathbb{E}\left[Y_{i}(\phi) Y_{j}(\phi) I_{2 r-2(u+v)}\left(\partial_{\frac{i}{n}}^{\otimes r-2 u} \widetilde{\otimes} \partial_{\frac{j}{n}}^{\otimes r-2 v}\right)\right]\right| \\
& +C \sum_{u, v=0}^{\left\lfloor\frac{r}{2}\right\rfloor} \sum_{z=1}^{(r-2 u) \wedge(r-2 v)} n^{-2 H(u+v)} \sum_{i, j=0}^{\lfloor n T\rfloor-1} \mid \mathbb{E}\left[Y_{i}(\phi) Y_{j}(\phi)\right. \\
& \left.\times I_{2 r-2(u+v)-2 z}\left(\partial_{\frac{i}{m}}^{\otimes r-2 u-z} \widetilde{\otimes} \partial_{\frac{j}{n}}^{\otimes r-2 v-z}\right)\left\langle\partial_{\frac{i}{n}}, \partial_{\frac{j}{n}}\right\rangle_{\mathfrak{H}}^{z}\right] \mid \\
& =: C\left(D_{1}+D_{2}\right) \text {. }
\end{aligned}
$$

We first study term $D_{2}$, that is when $z \geq 1$. On one hand, from the estimate (2.5), we get

$$
\begin{aligned}
& \left\|I_{2 r-2(u+v)-2 z}\left(\partial_{\frac{i}{n}}^{\otimes r-2 u-z} \widetilde{\otimes} \partial_{\frac{j}{n}}^{\otimes r-2 v-z}\right)\right\|_{L^{q /(q-2)}(\Omega)} \leq C\left(\left\|\partial_{\frac{i}{n}}\right\|_{\mathfrak{H}}^{r-2 u-z}\left\|\partial_{\frac{j}{n}}\right\|_{\mathfrak{H}}^{r-2 v-z}\right) \\
& =C\left\|\partial_{\frac{1}{n}}\right\|_{\mathfrak{H}}^{2 r-2(u+v)-2 z} \\
& =C n^{-2 H(r-u-v-z)} \text {. }
\end{aligned}
$$


On the other hand, using (2.10), we obtain

$$
\sum_{i, j=0}^{\lfloor n T\rfloor-1}\left|\left\langle\partial_{\frac{i}{n}}, \partial_{\frac{j}{n}}\right\rangle_{\mathfrak{H}}^{z}\right| \leq C n^{1-2 z H} .
$$

Thus, from (A.5) and (A.6) and using Hölder's inequality, we deduce that the term $D_{2}$ is bounded by

$$
\begin{aligned}
D_{2} \leq & C \sum_{u, v=0}^{\left\lfloor\frac{r}{2}\right\rfloor} \sum_{z=1}^{(r-2 u) \wedge(r-2 v)} n^{-2 H(u+v)} \\
& \times \sup _{0 \leq j \leq\lfloor n T\rfloor-1}\left\|Y_{j}(\phi)\right\|_{L^{q}(\Omega)}^{2} n^{-2 H(r-u-v-z)} n^{1-2 z H} \\
\leq & C \sup _{0 \leq j \leq\lfloor n T\rfloor-1}\left\|Y_{j}(\phi)\right\|_{L^{q}(\Omega)}^{2} n^{1-2 r H} .
\end{aligned}
$$

Now, let us study term $D_{1}$, that is, when $z=0$. By (2.6), we have

$$
\begin{aligned}
& \left|\mathbb{E}\left[Y_{i}(\phi) Y_{j}(\phi) I_{2(r-u-v)}\left(\partial_{\frac{i}{n}}^{\otimes r-2 u} \widetilde{\otimes} \partial_{\frac{j}{n}}^{\otimes r-2 v}\right)\right]\right| \\
& \quad=\left|\mathbb{E}\left[\left\langle D^{2(r-u-v)}\left(Y_{i}(\phi) Y_{j}(\phi)\right), \partial_{\frac{i}{n}}^{\otimes r-2 u} \widetilde{\otimes} \partial_{\frac{j}{n}}^{\otimes r-2 v}\right\rangle_{\mathfrak{H}^{\otimes 2(r-u-v)}}\right]\right| .
\end{aligned}
$$

Write $s=2(r-u-v)$. By definition of Malliavin derivative and Leibniz rule, $D_{u_{1}, \ldots, u_{s}}^{s}\left(Y_{i}(\phi) Y_{j}(\phi)\right)$ consists of terms of the form $D_{\mathbf{u}_{J}}^{|J|}\left(Y_{i}(\phi)\right) D_{\mathbf{u}_{J^{c}}}^{s-|J|}\left(Y_{j}(\phi)\right)$, where $J$ is a subset of $\{1, \ldots, s\},|J|$ denotes the cardinality of $J$ and $\mathbf{u}_{J}=\left(u_{i}\right)_{i \in J}$. Without loss of generality, we may fix $J$ and assume that $a=|J| \geq 1$. By our assumptions on $\phi$ and the definition of Malliavin derivative, we know that

$$
\begin{aligned}
D^{a}\left(Y_{i}(\phi)\right) & =\phi^{(a)}\left(\widetilde{B}_{\frac{i}{n}}^{H}\right) \widetilde{\varepsilon}_{\frac{i}{n}}^{\otimes a}-\phi^{(a)}\left(B_{\frac{k(i)}{m}}^{H}\right) \varepsilon_{\frac{k(i)}{m}}^{\otimes a} \\
& =Y_{i}\left(\phi^{(a)}\right) \varepsilon_{\frac{k(i)}{m}}^{\otimes a}+\phi^{(a)}\left(\widetilde{B}_{\frac{i}{n}}^{H}\right)\left(\widetilde{\varepsilon}_{\frac{i}{n}}^{\otimes a}-\varepsilon_{\frac{k(i)}{m}}^{\otimes a}\right),
\end{aligned}
$$

where we recall that $k=k(i)=\sup \left\{j: \frac{j}{m} \leq \frac{i}{n}\right\}$, and, for each $a \leq 2 r$, we have $D^{a}\left(Y_{i}(\phi)\right) \in L^{2}\left(\Omega ; \mathfrak{H}^{\otimes a}\right)$. Setting $b=s-|J|=s-a$ and with a slight abuse of notation, it follows that

$$
\begin{aligned}
\mathbb{E}\left[\left\langleD_{\mathbf{u}_{J}}^{a}(\right.\right. & \left.\left.\left.Y_{i}(\phi)\right) D_{\mathbf{u}_{J^{c}}}^{b}\left(Y_{j}(\phi)\right), \partial_{\frac{i}{n}}^{\otimes r-2 u} \otimes \partial_{\frac{j}{n}}^{\otimes r-2 v}\right\rangle_{\mathfrak{H}^{\otimes 2 r-2(u+v)}}\right] \\
\leq & \left\|Y_{i}\left(\phi^{(a)}\right)\right\|_{L^{2}(\Omega)}\left\|Y_{j}\left(\phi^{(b)}\right)\right\|_{L^{2}(\Omega)} \\
& \left.\times \| \varepsilon_{\frac{k(i)}{m}}^{\otimes a}\left(\mathbf{u}_{J}\right) \otimes \varepsilon_{\frac{k(j)}{m}}^{\otimes b}\left(\mathbf{u}_{J^{c}}\right), \partial_{\frac{i}{n}}^{\otimes r-2 u} \otimes \partial_{\frac{j}{n}}^{\otimes r-2 v}\right\rangle_{\mathfrak{H}^{\otimes \mathfrak{s}}} \mid \\
& +\left\|Y_{i}\left(\phi^{(a)}\right)\right\|_{L^{2}(\Omega)}\left\|\phi^{(b)}\left(\widetilde{B}_{\frac{j}{n}}^{H}\right)\right\|_{L^{2}(\Omega)} \\
& \times\left|\left\langle\varepsilon_{\frac{k(i)}{m}}^{\otimes a}\left(\mathbf{u}_{J}\right) \otimes\left(\widetilde{\varepsilon}_{\frac{j}{n}}^{\otimes b}-\varepsilon_{\frac{k(j)}{m}}^{\otimes b}\right)\left(\mathbf{u}_{J^{c}}\right), \partial_{\frac{i}{n}}^{\otimes r-2 u} \otimes \partial_{\frac{j}{n}}^{\otimes r-2 v}\right\rangle_{\mathfrak{H}^{\otimes \mathfrak{F}}}\right|
\end{aligned}
$$




$$
\begin{aligned}
& +\left\|\phi^{(a)}\left(\widetilde{B}_{\frac{i}{m}}^{H}\right)\right\|_{L^{2}(\Omega)}\left\|Y_{j}\left(\phi^{(b)}\right)\right\|_{L^{2}(\Omega)} \\
& \times\left|\left\langle\left(\widetilde{\varepsilon}_{\frac{i}{m}}^{\otimes a}-\varepsilon_{\frac{k(i)}{n}}^{\otimes a}\right)\left(\mathbf{u}_{J}\right) \otimes \varepsilon_{\frac{k(j)}{n}}^{\otimes b}\left(\mathbf{u}_{J^{c}}\right), \partial_{\frac{i}{m}}^{\otimes r-2 u} \otimes \partial_{\frac{j}{m}}^{\otimes r-2 v}\right\rangle_{\mathfrak{H}^{\otimes \mathfrak{s}}}\right| \\
& +\left\|\phi^{(a)}\left(\widetilde{B}_{\frac{i}{n}}^{H}\right)\right\|_{L^{2}(\Omega)}\left\|\phi^{(b)}\left(\widetilde{B}_{\frac{j}{n}}^{H}\right)\right\|_{L^{2}(\Omega)} \\
& \times\left|\left\langle\left(\widetilde{\varepsilon}_{\frac{i}{n}}^{\otimes a}-\varepsilon_{\frac{k(i)}{m}}^{\otimes a}\right)\left(\mathbf{u}_{J}\right) \otimes\left(\widetilde{\varepsilon}_{\frac{j}{n}}^{\otimes b}-\varepsilon_{\frac{k(j)}{m}}^{\otimes b}\right)\left(\mathbf{u}_{J^{c}}\right), \partial_{\frac{i}{n}}^{\otimes r-2 u} \otimes \partial_{\frac{j}{n}}^{\otimes r-2 v}\right\rangle_{\mathfrak{H}^{\otimes \mathfrak{F}}}\right| \\
= & : D_{11}+D_{12}+D_{13}+D_{14} .
\end{aligned}
$$

Consider first the term $D_{11}$. By (2.8), we have either

$$
D_{11} \leq C\left|\left\langle\varepsilon_{\frac{k(i)}{m}}, \partial_{\frac{j}{n}}\right\rangle_{\mathfrak{H}}\right| n^{-2 H(a+b-1)} \sup _{0 \leq w \leq 2 r} \sup _{0 \leq j \leq\lfloor n T\rfloor-1}\left\|Y_{j}\left(\phi^{(w)}\right)\right\|_{L^{2}(\Omega)}^{2}
$$

or

$$
D_{11} \leq C\left|\left\langle\varepsilon_{\frac{k(i)}{m}}, \partial_{\frac{i}{n}}\right\rangle_{\mathfrak{H}}\right| n^{-2 H(a+b-1)} \sup _{0 \leq w \leq 2 r} \sup _{0 \leq j \leq\lfloor n T\rfloor-1}\left\|Y_{j}\left(\phi^{(w)}\right)\right\|_{L^{2}(\Omega)}^{2} .
$$

By Lemma 2.1.a,

$$
\sum_{j=0}^{\lfloor n T\rfloor-1}\left|\left\langle\varepsilon_{\frac{k(i)}{m}}, \partial_{\frac{j}{n}}\right\rangle_{\mathfrak{H}}\right| \leq C
$$

and by (2.11),

$$
\sum_{i=0}^{\lfloor n T\rfloor-1} \sum_{j=0}^{\lfloor n T\rfloor-1}\left|\left\langle\varepsilon_{\frac{k(i)}{m}}, \partial_{\frac{i}{n}}\right\rangle_{\mathfrak{H}}\right|\left|\left\langle\varepsilon_{\frac{k(j)}{m}}, \partial_{\frac{j}{n}}\right\rangle_{\mathfrak{H}}\right| \leq C_{T} m^{2-4 H} .
$$

As a consequence, inequalities (A.7) and (A.8) imply

$$
\begin{aligned}
& \sum_{u, v=0}^{\left\lfloor\frac{r}{2}\right\rfloor} n^{-2 H(u+v)} \sum_{i, j=0}^{\lfloor n T\rfloor-1} D_{11} \\
& \leq C \sup _{0 \leq w \leq 2 r} \sup _{0 \leq j \leq\lfloor n T\rfloor-1}\left\|Y_{j}\left(\phi^{(w)}\right)\right\|_{L^{2}(\Omega)}^{2} \sum_{u, v=0}^{\left\lfloor\frac{r}{2}\right\rfloor} \sum_{i=0}^{\lfloor n T\rfloor-1} n^{-2 H(u+v+a+b-1)} \\
& \quad+C_{T} \sup _{0 \leq w \leq 2 r} \sup _{0 \leq j \leq\lfloor n T\rfloor-1}\left\|Y_{j}\left(\phi^{(w)}\right)\right\|_{L^{2}(\Omega)}^{2} \\
& \quad \times \sum_{u, v=0}^{\left\lfloor\frac{r}{2}\right\rfloor} n^{-2 H(u+v+a+b-1)} n^{2 H} m^{2-4 H} \\
& \leq C_{T} \sup _{0 \leq w \leq 2 r} \sup _{0 \leq j \leq\lfloor n T\rfloor-1}\left\|Y_{j}\left(\phi^{(w)}\right)\right\|_{L^{2}(\Omega)}^{2}\left(1+n^{1-2 H} m^{2-4 H}\right) n^{1-2 r H}
\end{aligned}
$$


where we used that $u+v+a+b-1=2 r-(u+v)-1 \geq r$, since $u+v+1 \leq$ $2\left\lfloor\frac{r}{2}\right\rfloor+1=r$ for any odd integer $r$.

We apply the same calculation to $D_{12}$ and $D_{13}$, and we similarly obtain that

$$
\begin{aligned}
& \sum_{u, v=0}^{\left\lfloor\frac{r}{2}\right\rfloor} n^{-2 H(u+v)} \sum_{i, j=0}^{\lfloor n T\rfloor-1}\left(D_{12}+D_{13}\right) \\
& \leq C_{T} \sup _{0 \leq w \leq 2 r} \sup _{0 \leq j \leq\lfloor n T\rfloor-1}\left\|\phi^{(w)}\left(\widetilde{B}_{\frac{j}{n}}\right)\right\|_{L^{2}(\Omega)} \sup _{0 \leq j \leq\lfloor n T\rfloor-1}\left\|Y_{j}\left(\phi^{(w)}\right)\right\|_{L^{2}(\Omega)} \\
& \quad \times\left(1+n^{2 H-1} m^{2-4 H}\right) n^{1-2 r H} .
\end{aligned}
$$

Now we study term $D_{14}$. Inequalities (2.12) and (2.13) state that

$$
\begin{gathered}
\sum_{j=0}^{\lfloor n T\rfloor-1}\left|\left\langle\widetilde{\varepsilon}_{\frac{i}{n}}-\varepsilon_{\frac{k(i)}{m}}, \partial_{\frac{j}{n}}\right\rangle_{\mathfrak{H}}\right| \leq C_{T} m^{-2 H} \text { and } \\
\sum_{i=0}^{\lfloor n T\rfloor-1}\left|\left\langle\widetilde{\varepsilon}_{\frac{i}{n}}-\varepsilon_{\frac{k(i)}{m}}, \partial_{\frac{i}{n}}\right\rangle_{\mathfrak{H}}\right| \leq C_{T} m^{1-2 H} .
\end{gathered}
$$

Then, with the same arguments as those used for $D_{11}$, we obtain

$$
\begin{aligned}
& \sum_{u, v=0}^{\left\lfloor\frac{r}{2}\right\rfloor} n^{-2 H(u+v)} \sum_{i, j=0}^{\lfloor n T\rfloor-1} D_{14} \\
& \quad \leq C_{T} \sup _{0 \leq w \leq 2 r} \sup _{0 \leq j \leq\lfloor n T\rfloor-1}\left\|\phi^{(w)}\left(\widetilde{B}_{\frac{j}{n}}^{H}\right)\right\|_{L^{2}(\Omega)}^{2}\left(m^{-2 H}+n^{2 H-1} m^{2-4 H}\right) n^{1-2 r H} .
\end{aligned}
$$

The proof is now complete.

Acknowledgments. We would like to thank the Associate Editor and the referees for a careful reading of our manuscript, and for several valuable comments.

\section{REFERENCES}

[1] Burdzy, K. and SWAnson, J. (2010). A change of variable formula with Itô correction term. Ann. Probab. 38 1817-1869. MR2722787

[2] Cheridito, P. and Nualart, D. (2005). Stochastic integral of divergence type with respect to fractional Brownian motion with Hurst parameter $H \in(0,1 / 2)$. Ann. Inst. H. Poincaré Probab. Statist. 41 1049-1081. MR2172209

[3] Corcuera, J. M., Nualart, D. and Podolskij, M. (2014). Asymptotics of weighted random sums. Commun. Appl. Ind. Math. 6 e-486. MR3277312

[4] Corcuera, J. M., NuAlart, D. and Woerner, J. (2006). Power variation of some integral fractional processes. Bernoulli 12 713-735. MR2248234

[5] Gradinaru, M., Nourdin, I., Russo, F. and Vallois, P. (2005). $m$-order integrals and generalized Itô's formula: The case of a fractional Brownian motion with any Hurst index. Ann. Inst. H. Poincaré Probab. Statist. 41 781-806. MR2144234 
[6] Harnett, D. and NuAlart, D. (2012). Weak convergence of the Stratonovich integral with respect to a class of Gaussian processes. Stochastic Process. Appl. 122 3460-3505.

[7] Harnett, D. and Nualart, D. (2013). Central limit theorem for a Stratonovich integral with Malliavin calculus. Ann. Probab. 41 2820-2879. MR3112933

[8] Harnett, D. and Nualart, D. (2015). On Simpson's rule and fractional Brownian motion with $H=1 / 10$. J. Theoret. Probab. 28 1651-1688. MR3422945

[9] Nourdin, I. (2009). A change of variable formula for the 2D fractional Brownian motion of Hurst index bigger or equal to 1/4. J. Funct. Anal. 256 2304-2320. MR2498767

[10] Nourdin, I. and PeCCATI, G. (2012). Normal Approximations with Malliavin Calculus: From Stein's Method to Universality. Cambridge Univ. Press, Cambridge.

[11] Nourdin, I. and RÉVEILlaC, A. (2009). Asymptotic behavior of weighted quadratic variations of fractional Brownian motion: The critical case $H=1 / 4$. Ann. Probab. 372200 2230. MR2573556

[12] Nourdin, I., RÉveillac, A. and Swanson, J. (2010). The weak Stratonovich integral with respect to fractional Brownian motion with Hurst parameter 1/6. Electron. J. Probab. 15 2117-2162. MR2745728

[13] Nualart, D. (2006). The Malliavin Calculus and Related Topics, 2nd ed. Springer, Berlin. MR2200233

[14] Nualart, D. and Ortiz-Latorre, S. (2008). Central limit theorems for multiple stochastic integrals and Malliavin calculus. Stochastic Process. Appl. 118 614-628. MR2394845

G. BinOTTO

FACUlTAT DE MATEMÀtiques

UNIVERSITAT DE BARCELONA

GRAN Via DE LES CORTS CATALANES 585

08007 BARCELONA

SPAIN

E-MAIL: gbinotto@ub.edu
I. NOURDIN

UNIVERSITÉ DU LUXEMBOURG

UNITÉ DE RECHERCHE EN MATHÉMATIQUES

MAISON DU NOMBRE

6 AVENUE DE LA FONTE

L-4364 ESCH-SUR-ALZETTE

GRAND DUCHY OF LUXEMBOURG

E-MAIL: ivan.nourdin@uni.lu

D. NUALART

DEPARTMENT OF MATHEMATICS

UNIVERSITY OF KANSAS

LAWRENCE, KANSAS 66045

USA

E-MAIL: nualart@ku.edu 